\title{
Development of a Test Facility for Air Revitalization Technology Evaluation
}

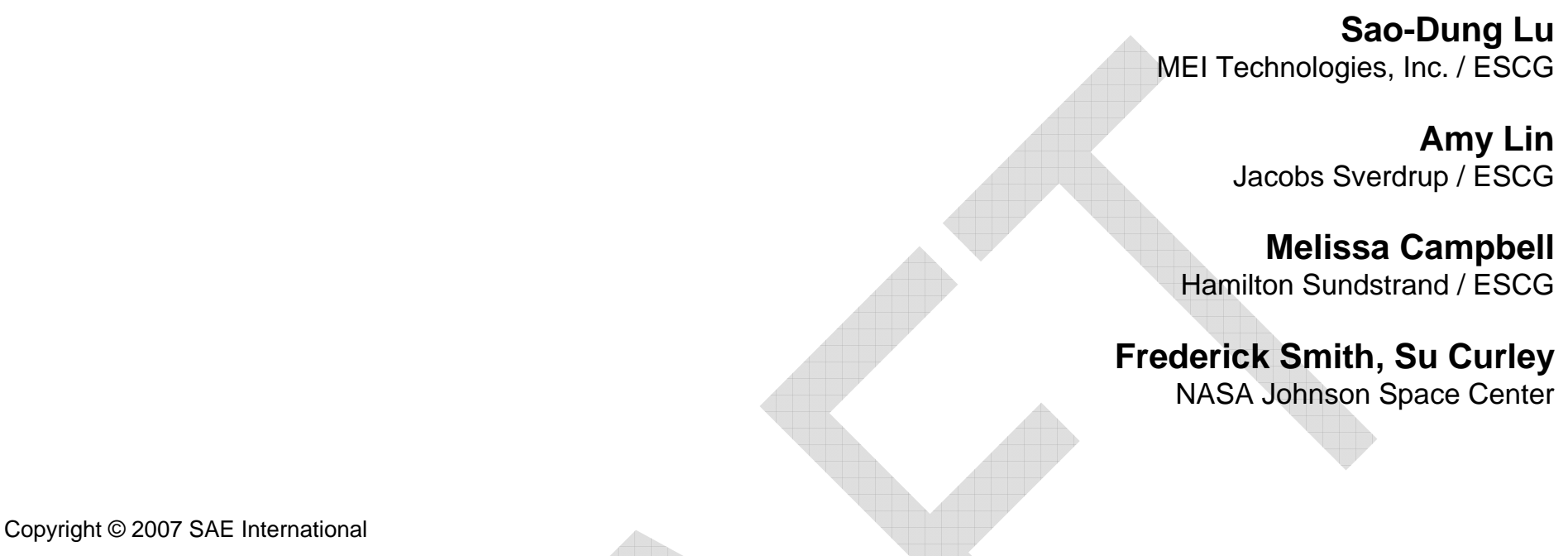

\begin{abstract}
Development of new air revitalization system (ARS) technology can initially be performed in a subscale laboratory environment, but in order to advance the maturity level, the technology must be tested in an endto-end integrated environment. The Air Revitalization Technology Evaluation Facility (ARTEF) at the NASA Johnson Space Center serves as a ground test bed for evaluating emerging ARS technologies in an environment representative of spacecraft atmospheres. At the center of the ARTEF is a hypobaric chamber which serves as a sealed atmospheric chamber for closed loop testing. A Human Metabolic Simulator (HMS) was custom-built to simulate the consumption of oxygen, and production of carbon dioxide, moisture and heat of up to eight persons. A multitude of gas analyzers and dew point sensors are used to monitor the chamber atmosphere upstream and downstream of a test article. A robust vacuum system is needed to simulate the vacuum of space. A reliable data acquisition and control system is required to connect all the subsystems together. This paper presents the capabilities of the integrated test facility and some of the issues encountered during the integration.
\end{abstract}

\section{INTRODUCTION}

Atmospheric revitalization is a discipline within the Environmental Control and Life Support (ECLS) which addresses the process technologies and equipment necessary to provide, monitor, and control the atmospheric environment within a crewed spacecraft or surface habitat cabin. This includes the capability to remove contaminants and condition cabin atmosphere, supply and store atmospheric gases, and closed loop processes which recycle resources. Processes include separation by physical adsorption, absorption, and mechanical filtration. Other processes may include chemical adsorption, reduction, and oxidation.

Lithium hydroxide and charcoal have been the dominant technology used to scrub the cabin atmosphere for decades. This open loop technology is so simple and reliable that no significant changes have been necessary. Until the International Space Station (ISS), the mission durations have been short. In order to develop new regenerative technology, a test facility must be able to sufficiently simulate a spacecraft atmosphere in order to aid in the development of the technology.

A survey was taken of existing facilities to evaluate their capabilities, strengths and weaknesses. Desired capabilities and design goals were established for an inhouse facility. This paper presents some of the issues and lessons learned in the process.

\section{FACILITIES SURVEY}

The Johnson Space Center contains numerous environmental chambers of all sizes. During a survey of these facilities, it was discovered that they did not meet our requirements. For the most part, they were either too small, too big, not sufficiently air tight, or incapable of being man rated. Various project managers were also 
interviewed to understand their capabilities, limitations, and lessons learned.

The result of the survey was that all existing facilities were already in full use and would not be able to support activities conceptualized for the ARTEF. The result of the analysis was the selection of a facility containing a hypobaric chamber that was formerly used as a plant growth laboratory. This space is also adjacent to the water recovery lab and thus would facilitate full scale integration activities.

The hypobaric chamber was named as the Air Revitalization Technology Integration Chamber (ARTIC). This chamber contains two air tight compartments, a main chamber and an airlock. The airlock and main chamber sections can have multiple uses in technology evaluation testing. Initially the airlock would be used as a mixing volume for various test articles. The long term goal was for the chamber to be human rated for extended duration testing.

As a future option, the ARTEF could also support continuous long-duration testing of bioregenerative life support systems. The ARTEF could operate in a manner capable of sustaining continuous test operations for extended periods. The first set of tests were planned to consist of shorter durations in order to mature the facility and test article systems.

\section{DESIGN GOALS}

The ARTEF must accommodate technology ranging from bench top breadboard type concept to a full scale proven technology ready to go into a flight hardware packaging. Needed to accommodate level of bench level testing as well as fully integrated testing with other related systems such as water recovery and plants. Representing a fully integrated closed loop recovery system.

We recognized that in order to adequately develop any new technology, we needed to have the capability to provide a flight like atmospheric environment. This includes the capability to simulate the human metabolic process of consuming oxygen, and producing carbon dioxide, heat and moisture. This would have to be capable of reproducing different number of crew members at different activity levels. Additionally, we need a sealed airtight environmental chamber, and sufficient instrumentation to monitor the environmental conditions inside the chamber. Including reduced pressure and elevated oxygen levels.

Typical operation of the evaluation lab would be to bench test each component of a technology to verify proper operation and document a baseline reference. Evaluations could also include control systems and endto-end systems. Individual and integrated components could be evaluated for short term and long term testing. Each component could be integrated with other components and tested again on a system level. Testing could occur in stages, with each stage involving a higher level of fidelity and metabolic loading. Initial metabolic loads would be simulated with the Human Metabolic Simulator (HMS). The last stages of testing could involve actual human metabolic loads.

Typical technologies include regenerable carbon dioxide removal, carbon dioxide collection and storage, carbon dioxide reduction to water, trace contaminant control, and environmental monitoring systems.

The ARTEF would be capable of conducting multiple simultaneous tests. For example, smaller component level testing may be performed while a long term test is under way in an integrated systems test bed. The first year of development was spent in requirements definition and facility survey.

NASA regulations require all systems to be failsafe. Critical systems are required to have redundant safety.

A list of required functions is given below:

- facility supply of gases: air, nitrogen, oxygen, carbon dioxide

- leak-tight environmental chamber (man-rated)

- facility available power, 120VAC, 208VAC, 480VAC in single and three phase

- 120 VDC power for ISS applications

- Chilled water cooling system

- liquid nitrogen supply

- room temperature control

- deionized water and drainage

- facility vacuum

- combustible venting to outside

- data acquisition and control network

- gas analyzer / mass spectrometer

- integration capability with water recovery systems plant growth systems, and thermal control systems. 


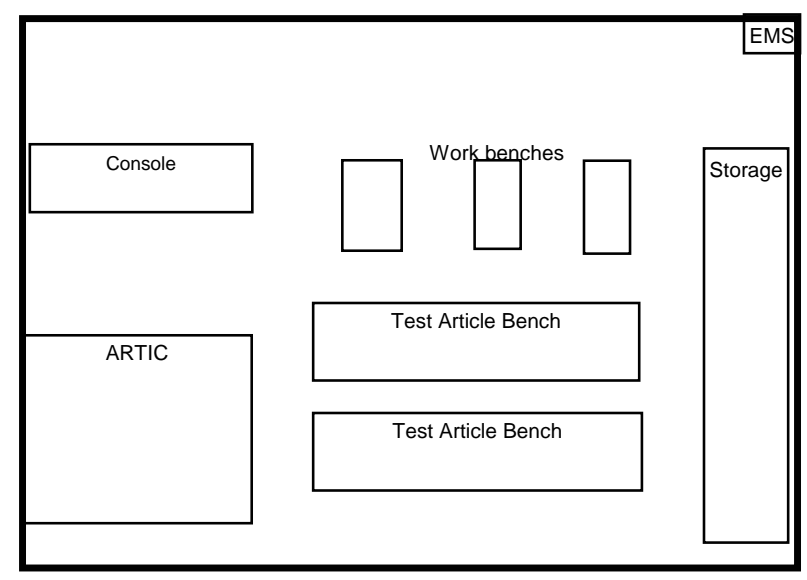

Figure 1. Layout of the ARTEF

\section{CABIN CHAMBER}

A leak tight chamber is needed to simulate a spacecraft cabin. The chamber serves as a mixing volume for the atmospheric gases. Having the right volume provides an indication of the time delay between the time the atmosphere changes and when the processing occurs. As such, it's necessary to have a variable volume to adjust to the needs of each test. Boxes were made up to be airtight space fillers. Mylar was the obvious choice material.

In order to maintain mass balance in the system, the chamber must be relatively tight. The ARTIC chamber was designed as an altitude chamber, which means that the door seals are designed to minimize leakage when the internal volume is at sub-ambient pressure.

Leakage testing was performed by filling the chamber with carbon dioxide and measuring the decay rate with a mass spectrometer, as well as a NDIR $\mathrm{CO}_{2}$ gas analyzer. The total pressure inside the chamber was initially equalized to ambient. Since the classic pressure decay curve is exponential, it will be linear when plotted on a logarithmic scale. This also means for the clearest results, relatively high concentrations of trace gas are needed and/or a long period of time. Neither was available here. Safety was nervous about high $\mathrm{CO}_{2}$ concentrations in case anyone inadvertently opened the door, and walked into the chamber. The team did not have the luxury of waiting 3-4 weeks for a precise decay measurement. Given the instrument accuracy, and the experimental accuracy required, the leak decay was measured over 5 days to get acceptable results.

\section{HUMAN METABOLIC SIMULATOR}

While testing with human subjects is ultimately required in such development, initial testing is generally conducted using equipment that simulates effects of human respiration, perspiration and metabolism on an enclosed atmosphere. The use of such equipment allows new technology to be tested and verified prior to human testing, including behavior under off-nominal and emergency conditions. A wide range of simulated crew activity profiles can also be evaluated and reproduced using a HMS. The HMS has proven to be the most complex and difficult to control processes. The design concept has been previously presented at ICES (Jeng, 20xx), and can be referenced for further detail. issues that have developed during the use of the design will be presented here.

A HMS at a minimum is required to consume/remove oxygen, produce $\mathrm{CO}_{2}$, moisture and sensible metabolic heat for multiple people and various metabolic rates all the while maintaining a mass balance with minimal external leakage. As optional capabilities it should accommodate reduced pressure, elevated oxygen atmosphere, and trace gases. It should not remove anything else from the atmosphere except for oxygen $\left(\mathrm{O}_{2}\right)$.

Daily-average metabolic consumption and production rates can vary considerably. Of the total metabolic heat production, part is dissipated by vaporization of water (latent heat), and part is dissipated by sensible heat transfer to the environment. In volumetric (or molar) terms, the ratio of $\mathrm{CO}_{2}$ production to $\mathrm{O}_{2}$ consumption is referred to as the respiratory quotient. This ratio varies little with activity level except during severe exercise. A constant respiratory quotient of 0.87 was assumed in Table 1. In addition to the primary effects discussed above, humans also contribute to the production (and possible consumption) of trace contaminants in the atmosphere, but this is typically not accounted for in the design of a HMS.

Table 1. Metabolic Load Design Points

\begin{tabular}{|l|c|c|c|c|}
\hline Case & $\begin{array}{c}\text { Steam } \\
\text { rate } \\
\mathrm{ml} / \mathrm{min}\end{array}$ & $\mathrm{O}_{2} \mathrm{slm}$ & $\mathrm{N}_{2} \mathrm{slm}$ & $\begin{array}{c}\mathrm{CO}_{2} \\
\mathrm{slm}\end{array}$ \\
\hline & & & & \\
\hline 1 CREW MEMBER & 1.3 & 0.46 & 0.052 & 0.41 \\
\hline $\begin{array}{l}\text { 1/2 CREW 3 NOM 1 } \\
\text { EXERCISE }\end{array}$ & 10.5 & 2.5 & 0.28 & 2.2 \\
\hline $\begin{array}{l}\text { FULL 8 CREW, } \\
\text { 1 EXERCISE }\end{array}$ & 14.8 & 4.3 & 0.48 & 3.7 \\
\hline $\begin{array}{l}\text { 1 CREW MEMBER } \\
\text { SLEEP }\end{array}$ & 0.53 & 0.2 & 0.022 & 0.17 \\
\hline
\end{tabular}

A survey of various methods of oxygen consumption and carbon dioxide production were investigated. The typical methods are:

- Catalytic combustion of fuel

- Hydrogen Fuel Cell

- Chemical oxygen scavenger

- Air bleed with membrane recovery

- Molecular sieve oxygen concentrator 
The molecular sieve oxygen concentrator (OC) was chosen for low temperature operation, and best retention of trace contaminants. A COTS oxygen concentrator was bought which was designed for medical use. Due to not understanding the operating principles, the OC was improperly installed. Due to the HMS system configuration, the downstream desorption pressure of the OC was too high, and prevented proper operation of the pressure swing cycling. The sorbent bed quickly became saturated with moisture. The $O C$ requires a pressure swing of at least 20 psid, and the desorption pressure must $<=14.7$ psia.

One of the main issues with initial design was being able to get sufficient heat energy into the process flow. Based on vendor data, shell and tube compact heat exchangers were selected which turned out to be greatly under sized by $50 \%$. Due to insufficient $\mathrm{HX}$ sizing, the hot oil bath (heat source) temperature was incrementally raised until satisfactory results were achieved. This caused the open hot oil bath to off gas hydrocarbons and cause the oil to experience accelerated oxidization. Better temperature control with properly sized $\mathrm{HX}$ would extend the service life of the heating oil.

Maintaining steam quality was most difficult. Given the relatively low moisture levels, a minimal amount of heat was needed for the latent heat of vaporization. In the liquid phase, the water injection rate varied from 0.5 to $15 \mathrm{ml} / \mathrm{min}$. This injection rate is not much more than a bubbler provides. But this proved to be unsuccessful. The steam must be slightly super-heated prior to adiabatic expansion into the process flow stream. The water boiler needed to be carefully controlled using a combination of temperature and pressure to maintain a slightly super-heated state. The pressure, and then consequently the temperature is controlled by a metering valve. The opening of the metering valve was dependant upon the flow rate and the wet steam pressure.

Handling of steam and moist air is the most troublesome in all systems. Regardless of system, the moisture tends to stick to the tubing walls, causing a significant lag in the humidity and response time. The system is also prone to condensation. Even with much additional heat trace in the piping, results were not as good as desired. This was the case in the whole length of the process line returning to the chamber.

\section{ENVIRONMENTAL MONITORING}

During the initial build up of the ARTEF, numerous Gas Analyzer Consoles (GAC) were found which were not being utilized. Though these consoles had not be operational in years, they were successfully used in other chamber operational tests. These GACs were thought to have provided successful and accurate monitoring of various sealed chamber environments. These GACs were brought to the ARTEF for recertification. Ultimately, these GACs were problematic in that they leakage and other issues with the designed flow path.

Aside from the specific issues below, there were typical issues such as packaging and layout. Ideally a sensor or detector should be as close to the sample point as possible, providing minimal lag time in the reading, but ultimately not everything can fit directly adjacent to any test article. The need for remote sensing capabilities really came to light. If an instrument employed a remoter sensor probe, this greatly helped with the layout and packaging. But in some cases, a remote sensor probe is not available, and an atmospheric gas sample line must be employed. Gas analyzer consoles were developed to perform the environmental monitoring of carbon dioxide and humidity.

\section{CARBON DIOXIDE MONITORING}

Ideally, to minimize the response lag time, the gas analyzer must be placed as close to the process flow as possible. Due to the size requirements and limitations in our test chamber, the carbon dioxide analyzers had to be placed outside the chamber. Due to plumbing and other hardware, the analyzer ultimately ended up with a sample line length of almost 20 linear feet. With a $1 / 4 "$ diameter sample line, the response lag was 12 seconds with a 1 liter/min flow rate. But then add the time for homogenous mixing in the chamber, could be on the order of minutes.

Under most conditions, the small amount of humidity in the atmospheric sample can be ignored in the concentration of the carbon dioxide. However, their signatures are very close for the NDIR detection method. In this case, because there are significant spikes in humidity levels at times, a cold trap is required in the sample inlet line. The cold trap should be fed by a chiller cart in order to maintain a constant and repeatable dew point humidity level.

The sample lines should be of small diameter to minimize the amount of time it takes to reach the detector. But small diameter contributes to pressure drop line losses which must be compensated for by the sample pump. But the NDIR detector can only handle a limited flow rate and pressure. Ideally a large circulation flow rate is used to minimize the response lag, while a smaller slip stream is taken off for the detector.

Relatively large diaphragm pumps had previously been used along with a bypass loop to adjust the flow rates. Ultimately, the bypass loop itself caused a resonant pulse to occur inside the gas sample line, and caused poor flow rates through the $\mathrm{CO}_{2}$ analyzer and questionable data.

Due to the pressure limitations, relief valves must be employed to protect the system. Relief valves were found to be leaky and did not re-seat properly. These 
issues required the relief vent to be captured and returned to the chamber to pressure the mass balance.

\section{HUMIDITY MONITORING}

Accurate humidity sensing has proven to be most challenging. Chilled mirror technology is very common and accurate. It is widely, successfully used in most applications, but not in this case. This application is demanding due to the rapidly changing humidity levels experienced in various swing bed technology. Chilled mirrors were unable to have a fast enough rate of change response. Another shortcoming of the chilled mirror method is that a layer of ice will accumulate on the chilled mirror at the low dew points. Chilled mirror tends to be more stable, and best for slow changing or steady state conditions.

There are various other technologies using resistive or capacitance methods to measure humidity. Most of the devices only read relative humidity and not dew point. Aluminum oxide technology was found to have much faster response at the sacrifice of slightly lower accuracy. These are excellent at low dew points, but can become easily saturated at high dew points and need to be dried before reuse. These also tend to have more drift.

Regardless of the type of analyzer used, the amount of water vapor absorbed by the plumbing is enough to cause significant errors. Even if the moisture remains in the vapor phase, large amounts of moisture can be absorbed by metal lines, fittings, and valves. Due to the geometry and surface contamination, the water vapor can easily condense or evaporate in the lines, causing unexpected changes in humidity levels.

\section{ELECTRICAL POWER \& EMS}

Initial design specifications for the ARTEF were difficult to define due to the fact that no actual test articles had been built yet, and specific test requirements had not yet been defined. Estimates were made based on the number of test articles operating simultaneously, and the support equipment expected. The ARTEF power requirements were grossly undersized.

In order to accommodate so many different test articles and support equipment, many different flavors of power would also be necessary. These included the typical 110 VAC, 208 VAC single and 3 phase, including 480 VAC single and 3 phase. In anticipation of ISS targeted applications, 120VDC is also available.

As a measure of configuration control, the electricians used various twist-lock plugs and receptacles so that only the designated appliances could be plugged into these receptacles, preventing occasional use of these receptacles for any other appliances or articles. This proved to be terribly inconvenient.
Additionally, the size and location of the power and lighting panels were considered. This includes the total ampacity of the room, how power is distributed, and expansion flexibility and convenience. A 4" X 4" wireway is commonly used for convenience and flexibility. Due to the large number of wires, regular round conduit had to be derated in their ampaciity. Issues arose with wire insulation, and how many wires were within each conduit of each size.

Most test article circuits are Ground Fault Circuit Interrupted (GFCl). Due to large variety of appliances and breadboard level devices, extra levels of protection don't hurt even with extensive failsafe analysis

Since oxygen and hydrogen sources were also present, numerous additional safety measures are required. All electrical power needed to be on emergency power kill switching. All electrical power to the facility is disconnected via shunt trip breakers, in case of high ambient oxygen or hydrogen levels.

Prior to initiating the ARTEF buildup, it was determined that the facility employs a smoke and thermal detection EMS which reports to the Fire Panel on the North wall of the High Bay and the Emergency Operations Center $(E O C)$. There was also a sprinkler system that would be manually activated in case of a fire event. There were additional smoke and UV detectors inside the building 7 High Bay chambers that also reported to the Fire Panel and the EOC. Any event indicated at the Fire Panel would open the high bay door and activate all alarm bells, including the red alarm bell in the ARTEF.

The VPGC previously employed an independent smoke detection and power kill EMS, which was deactivated. Smoke detectors were to be installed in the ARTIC and tied to the building 7 Fire Panel. Due to the introduction of hydrogen and methane in the ARTEF, a system was required to detect elevated levels of combustible gases and initiate an automatic power kill to all items in the ARTEF. The ARTEF EMS has no connection with the building 7 Fire Panel.

Due to safety concerns associated with combustible gases, the ARTEF EMS cabinet was located near the primary exit door, far removed from the location of the test articles. Initial feedback from Johnson Space Center (JSC) Safety was that the EMS cabinet had to be in a separate room, with a solid fire-proof wall protecting it from the ARTEF. A compromise was finally reached due to the fact that the levels of gas are very low and the EMS cabinet was far away from the experiment area, with no flammables adjacent. Later, JSC Safety required additional measures of having indicators outside of the ARTEF to alert personnel prior to entering the room. Lights and a 'push-to-test' button were installed outside the door. The push-to-test button energized the lights and klaxon without alerting the EOC. The EMS designed for the ARTEF is for elevated hydrogen and oxygen detection only; however, the 
hydrogen sensor also detects methane. If elevated levels of hydrogen or oxygen are detected, the hydrogen and oxygen gas supply is shut off, ARTEF power circuits are opened and the EOC is alerted. The ARTEF employs redundant hydrogen and oxygen sensors. The EMS cabinet also includes a manual power kill button on the front panel, so that the EMS could be triggered as the personnel are fleeing. The manual power kill button does not alert the EOC initially, but once the sensors lose power, the alarm will trip and the EOC would be alerted.

\section{DATA ACQUISITION AND CONTROL NETWORK}

A trade study of the currently available data acquisition and control software was performed in order to upgrade the control and data acquisition systems throughout the JSC Crew and Thermal Systems Division (CTSD). Four of the major data acquisition and control systems were considered. Most of these were current versions of legacy systems in used at JSC and many other industrial sites. The study found an industry standard called Object Linked Executable (OLE) Process and Control (OPC) compliant hardware and software.

Ultimately, a tiered approach using OPC compliant components was selected. This offered the most flexibility and security as well as enhanced capabilities. This OPC compliant system was newly implemented in the ARTEF in conjunction with a in-house developed application package called 3T, which stands for a threetiered control hierarchy. This also became an opportunity to participate in some joint integration activities with the separate $3 T$ in-house developed control software application.

\section{INTEGRATED DISPLAY CONTROLLERS}

The initial design requirement for the HMS was to allow for automated computer control, as well as a fully manual front panel control. This would allow portability and versatility.

Many small COTS display \& control modules were bought which also allowed computer network control via a serial bus. In the end, these 18 modules have proven to be the cause of many of the controller communication problems. Given today's technology, front panel control would be better achieved by plugging in a laptop computer to a network interface on the equipment. This was meant to be the first tier control and data display. The ultimate design ended up with a number of items which are not controlled by these modules for a number of reasons. First, the team ran out of modules. Second, these components already had their own integrated display and control panels. Third and finally, the components did not have any data output, so these modules were not needed, e.g. SSR.
Programmable Logic Controllers (PLC) were used as the second tier control. These PLCs provide data acquisition and also allow the control software to run embedded in the PLC. This provides a faster processing, and also allows the equipment to continue to run, even if communication with the server is lost.

The next tier employs a COTS data acquisition and control application. This application software contains the correlation coefficients to convert the raw data signals to engineering units. The application provides a graphical user interface and control logic.

The third tier employs an OPC compliant human machine interface ( $\mathrm{HMI}$ ) which performs the data recording, provides the graphical data displays, and trend plots. This application allows the control and data display screens to be presented in HTML format. This means that any computer that can access the internet can be used to control and view the test data.

\section{VACUUM PUMP SYSTEM}

Most of the current regenerative carbon dioxide removal technology employs pressure swing sorbent beds. As such, a robust vacuum pumping train is required to simulate the vacuum of space. The system must accommodate high 'slugs' of carbon dioxide and moisture at regular intervals. The system must have sufficient capacity to move this volume of gas, and also to have very good ultimate vacuum levels. A sorbent bed is very sensitive to vacuum levels which can determine how well the bed desorbs between cycles. These vacuum pumps discussed here are not used to evacuate the chamber itself. All of the testing is currently be performed at 14.7 psia ambient pressure.

The pre-existing facilities turned out to be very unsatisfactory. Many of the pumps were too old and required refurbishment. They lacked the pumping rate and sufficient ultimate levels.

Initially a pair of large Beach Russ rotary vane pumps designed to evacuate large altitude chambers were used. Due to the possibility of high oxygen concentrations in the altitude chambers, these pumps are rated for oxygen compatibility. As a result, Fyrquel 550 is used in the pumps as a sealing/lubricating oil. High levels of moisture would quickly emulsify in the oil causing the ultimate vacuum levels to degrade rapidly.

The next attempts were to use a second set of large Beach Russ rotary vane pumps along with a large 8 foot diameter altitude chamber. The 8 foot chamber itself was also used as a vacuum plenum. This would help to minimize the effect of the large slugs every time a bed cycles. This proved to be an effective set up, although very expensive, time consuming and entailed additional 
scheduling constraints. Ultimately the high moisture rate also prove to be the downfall of this pumping train.

Most vacuum pumping trains in the CTSD have employed the used of liquid nitrogen cold traps. These vacuum pumps are rotary vane pumps which depend on oil as a sealing fluid and also as pump lubrication. In order to prevent the moisture from emulsifying into the oil, large liquid nitrogen cold traps are used upstream of the pumps. The cold traps come with the added overhead of replenishing the liquid nitrogen and melting/ draining of the condensate.

Ultimately, a new vacuum pumping train was purchased. After much research and consideration, it was decided to avoid the use of cold traps and to use pumps which could ingest significant amounts of moisture vapor. In addition to the issues previously mentioned, the cold traps also have leakage in the weld joints due to the cryogenic temperatures.

The new pumping train consists of a three stage pump system designed to provide a combined $2460 \mathrm{acfm}$. The first stage is an $1100 \mathrm{cfm}$ non-contacting rotary lobe blower with a variable frequency drive. The second stage is a $720 \mathrm{cfm}$ non-contacting rotary lobe blower. The final stage is a 180 acfm dry screw pump. The three pump stages are activated sequentially based on the upstream vacuum pressure. The dry screw pump is activated first, then the second stage blower, and lastly, the first stage blower is only activated when the pressure is below 3 torr. The large first stage blower speed varies according to the vacuum level desired.

The control of these three pumps is based on the upstream vacuum pressure required. Initially, the dry screw pump and the small blower pulls from 14.7 psia down to approximately 15 torr. Below this point, the first stage blower is activated to bring the vacuum down to an ultimate level of 0.5 torr.

\section{CONCLUSION}

Despite the wealth of skills and experience available in the CTSD, each application is different and entails a unique set of requirements and a unique configuration. With advancing technology in sensors, instruments and control systems, engineers desire to use the state of the art equipment. But with new technology, also comes a new learning curve.

Things seldom work as expected. Project schedule must include significant amount of time for functional checkout and troubleshooting. It is critical to fully understand the operating principles of various sensors and instruments. As well as the accuracy and precision of the sensor instruments. Sensors may not provide the desired data inputs.

Analytical tools should be fully utilized to the extent that they are available. Ideally, a mathematical model of new systems can help to identify issues before the hardware is built. An analytical model can help to identify whether adequate sensor instrumentation is designed into the system. Design of Experiment, or fractional factorial principles can help to maximize the results obtained from a minimum set of test points.

A healthy margin is also desired for testing. By the time the planned test cases are run, you realize that the data analysis would be greatly improved with just a couple additional test points.

\section{ACKNOWLEDGMENTS}

The authors acknowledge the contributions and support provided by the Crew and Thermal Systems Division (Fredrick Smith, Suzanne Curley and Dr. Jeff Sweterlitsch, Melissa Campbell, Amy Lin, Frank Jeng, Bruce Duffield, and Kevin Lange. None of this would have been possible without the staff of engineers and technicians who were responsible for building the facility: Mathew Blackmer, Kent Fry, Jason Pond, Josh Lotz.

\section{REFERENCES}

1. Lange, Kevin et al. ,Development of a Human Metabolic Simulator (HMS) for Air Revitalization System Testing, ICES 961523, July 2004

2. Duffield, Bruce et al, Redesign of the Human Metabolic Simulator, ICES 2004-01-2497

3. Jeng, Frank et al,

\section{ACRONYMS \& ABBREVIATIONS}

\begin{tabular}{llll} 
acfm & \multicolumn{2}{l}{ actual cubic feet per minute } \\
ARTEF & $\begin{array}{l}\text { Air Revitalization } \\
\text { Facility }\end{array}$ & Technology & Evaluation \\
ARTIC & $\begin{array}{l}\text { Air Revitalization } \\
\text { Chamber }\end{array}$ & Technology & Integration \\
cfm & $\begin{array}{l}\text { cubic feet per minute } \\
\text { CTSD }\end{array}$ & Crew \& Thermal Systems Division, JSC \\
JSC & NASA Johnson Space Center & \\
NASA & National Aeronautics and & Space \\
& $\begin{array}{l}\text { Administration } \\
\text { pounds per square inch absolute. }\end{array}$
\end{tabular}




\section{CONTACT}

Sao-Dung Lu.

ARS Project Manager

MEI Technologies, Inc.

Mailcode JE77/EC3

NASA Johnson Space Center

2101 NASA Pkwy.

Houston, TX 77058

Email: slu@ems.jsc.nasa.gov 